\title{
Is transthoracic ultrasound (TUS) a reliable predictor of the nature of pleural and peripheral pulmonary lesions? Correlation with cyto-histological findings
}

\author{
Mohamed H. Faheem
}

\begin{abstract}
Background: We focused on determining the most reliable transthoracic ultrasound (TUS) criteria that can predict malignancy of the pleural and peripheral pulmonary lesions and correlating our data with $C T$ and cyto-histological findings. Sixty-nine patients (38 males and 31 females) were enrolled in our prospective study. They were divided into group I (48 patients), which included patients with pleural effusion, and group II (21 patients), which included patients with peripheral pulmonary lesions. All patients underwent chest X-ray (postero-anterior and lateral views), transthoracic ultrasound (TUS), CT chest, guided Tru-cut or surgical biopsies and histopathology, and/or needle aspiration and cytology.
\end{abstract}

Results: In group I, the presence of pleural nodularity was a predictor of malignancy with significant statistical difference ( $b$ value $<0.001$ ) and receiver operating curve $(R O C)$ curve analysis showed pleural thickening cutoff value of $7 \mathrm{~mm}$ at a maximum combined sensitivity and specificity of $75 \%$ and $80 \%$ respectively and area under the curve 0.825. In group II, distorted intralesional vascular pattern, pleural, and chest wall invasion were statistically significant ultrasonographic malignant predictors ( $b$ values are $<0.001,0.016$, and 0.004 respectively).

Conclusion: TUS is a valuable and safe complementary method in differentiation between benign and malignant pleural effusion and peripheral pulmonary lesions but is not a substitute for CT.

Keywords: Transthoracic ultrasound, Thoracic malignancy

\section{Background}

The peripheral pulmonary and pleural lesions represent an important problem in the pulmonary diagnosis. They can remain undetermined after radiographic and CT scan analysis, so ultrasound can contribute in assessing the nature of these lesions [1].

The interest in transthoracic ultrasound (TUS) has increased after the portable ultrasound (US) equipments became suitable for the patient's bedside. TUS enables us to detect pleural and subpleural lung lesions, even in emergency settings [2]. Other advantages of TUS include easy personnel training, low cost, lack of radiation [3], and thus easy reevaluation [4].

Correspondence: Dr.hosny.rad@gmail.com

Department of Radiology, Banha Faculty of Medicine, Benha University

Hospital, Banha University, Farid Nada street, Banha, Qualuobyyia, Egypt
Although CT remains the main imaging modality in the diagnosis of the pleural and peripheral pulmonary lesions, however, Hallifax et al. found that the use of TUS is a valuable complementary imaging modality for evaluation of the pleural lesions [5].

Quershi et al. stated that pleural thickening $>1 \mathrm{~cm}$, pleural nodularity, and diaphragmatic thickening $>7 \mathrm{~mm}$ were highly suggestive ultrasound criteria of malignant pleural effusion [6]. Yilmaz et al. suggested pleural nodularity, bilateral pleural involvement, mediastinal pleural involvement, pleural rind, and pleural thickening $>1 \mathrm{~cm}$ as the CT predictors of malignancy [7]. Hierholzer et al. added chest wall and/or diaphragmatic infiltration as CT and MRI signs of malignancy, but they -in contradication to Quershi et al. - mentioned the pleural thickness 
$>1 \mathrm{~cm}$ as a nonsignificant factor in differentiating between benign and malignant pleural lesions [8].

In their prospective study, Abd El-hafez et al. found that pleural nodularity is not specific for the malignant lesions and more often seen with tuberculosis. They concluded that the oval and rounded shape is statistically significant for pleural and peripheral pulmonary malignant masses while the benign ones tend to be irregular [9]. Rednic and Orasan-unlike Abd El-hafez et al.-stated that the irregular pleural outline, destruction of normal tissue architecture, and vascular displacement are specific signs for malignancy [1].

In our study, we focused on determining the most reliable TUS criteria that can predict malignancy of the pleural and peripheral pulmonary lesions and correlated our findings with CT and cyto-histological findings. We compared our work with the available previous literature trying to validate the controversial points of their results.

Using TUS as a routine diagnostic tool in pleural and peripheral pulmonary lesions can help the treating physician in avoiding unnecessary further investigation in low-risk patients and to arrange high-yield diagnostic protocol for the high-risk ones [10].

\section{Patient and method}

Sixty-nine patients (38 males and 31 females) were enrolled in our prospective study, their ages ranged from $15-80$ years old (mean age $=47.4$ years), and they represented all adult patients whose initial chest X-ray showed pleural and/or peripheral pulmonary lesions and presented to the pulmonology and thoracic surgery departments in Banha University hospitals during the period from July 2016 to July 2017.

We excluded from the study the patients whose pulmonary lesions lacked adequate acoustic access; patients who underwent previous intervention outside our hospital; those who were not candidate for guided biopsy, needle aspiration, or surgical intervention (no pathological proof); and those who refused to sign informed consent.

The study sample was divided into two groups: group I (48 patients; 20 males and 28 females) included patients with pleural effusion, and group II (21 patients; 18 males and 3 females) included the patients with peripheral pulmonary lesions.

A written informed consent was taken from all the subjects after discussion of risks, considering the privacy of the patient. All the patients underwent the following: comprehensive history taking by the pulmonogist, initial chest X-ray (postero-anterior and lateral views), transthoracic ultrasound (TUS) examination, and CT scan of the chest (with or without IV contrast); nearly all the patients underwent guided Tru-cut or surgical biopsies and histopathology +/- needle aspiration and cytology (3 patients in group I underwent needle aspiration and cytology only).

\section{Transthoracic ultrasound technique}

All the TUS studies were carried out on "GE logiq P6 pro" ultrasound equipment using convex array (3-5 $\mathrm{MHz}$ ) and linear array $(4-11 \mathrm{MHz})$ probes according to the lesion site and nature. The lesion was localized first on the initial chest X-ray then examined in transverse and longitudinal planes using intercostal, subcostal, supra-, parasternal, or paravertebral accesses according to lesion location. The patient was examined in either supine, prone, lateral, or seated position depending on the area being examined and the patient comfort.

In group I (pleural effusion group), we commented on:

1. Pleural fluid echogenicity: According to Yang et al., it was classified into: anechoic (totally echo-free space was presented between the visceral and parietal pleura) (Fig. 1), complex nonseptated (echogenic materials were presented inside the effusion) (Fig. 2), complex septated (floating strands or septa were presented inside the effusion) (Fig. 3), and homogeneously echogenic (homogeneously echogenic pleural space was demonstrated) [11]

2. Volume: According to Tsai and Yang, the volume of the pleural effusion was quantified as follows:

minimal (the echo-free space was seen within the costophrenic angle), small (the space was seen over the costophrenic angle but still within a one-probe range), moderate (when seen within a two-probe range), and large or massive (when the space was bigger than a two-probe range) [12]

3. Presence of pleural thickening and nodularity (Figs. 2 and 3): When it was present, the maximum thickness and the dimensions of any focal pleural mass were measured

4. Presence of underlying pulmonary consolidation or masses

In group II (The peripheral pulmonary lesions group), we commented on:

1. Echogenicity: Hypoechoic, hyperechoic, or isoechoic to liver

2. Echopattern: Homogeneous, heterogeneous, heterogeneous +/- areas of breaking down or calcification

3. Presence of an air bronchogram

4. +/- Extension to pleura and chest wall: According to Sugama et al., the pleural invasion patterns were classified as the following: 

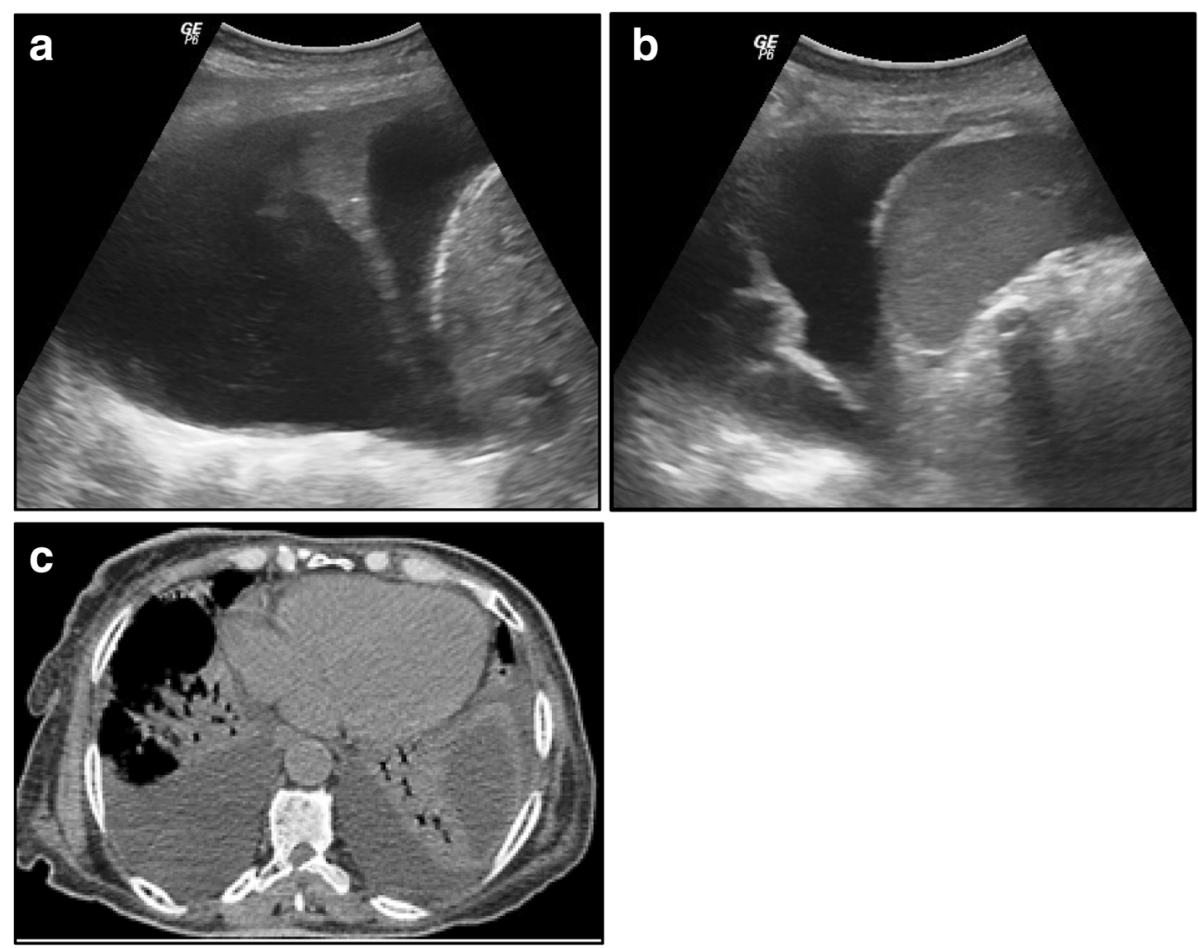

Fig. 1 US images show moderate right (a) and mild left (b) anechoic pleural effusion with underlying collapsed lung parenchyma. No evidence of pleural nodularity. $\mathbf{c} C T$ of the same patient revealed bilateral free pleural effusion with underlying compression collapse. Cytological examination of the pleural fluid revealed transudate

- UP (ultrasound pattern) 1 (indicates that the tumor is in contact with the visceral pleura): It shows intact visceral pleura and movement of the tumor with respiration

- UP2 (the tumor has extended beyond the visceral pleura and was in contact with the parietal pleura): It shows interrupted visceral pleura, disturbed tumor movement with respiration with no direct extension of the lesion to the chest wall
- UP3 (the tumor has extended to the chest wall through both visceral and parietal pleurae) (Fig. 4): It shows interrupted visceral pleura, absent tumor movement with respiration, and direct extension of the lesion to chest wall [13]

5. Vascularity by color duplex: Either pulmonary branching pattern of vascularity or distorted (either displaced vascularity, non-branching pattern of vascularity, absent or little vascularity, or vascular malformation)

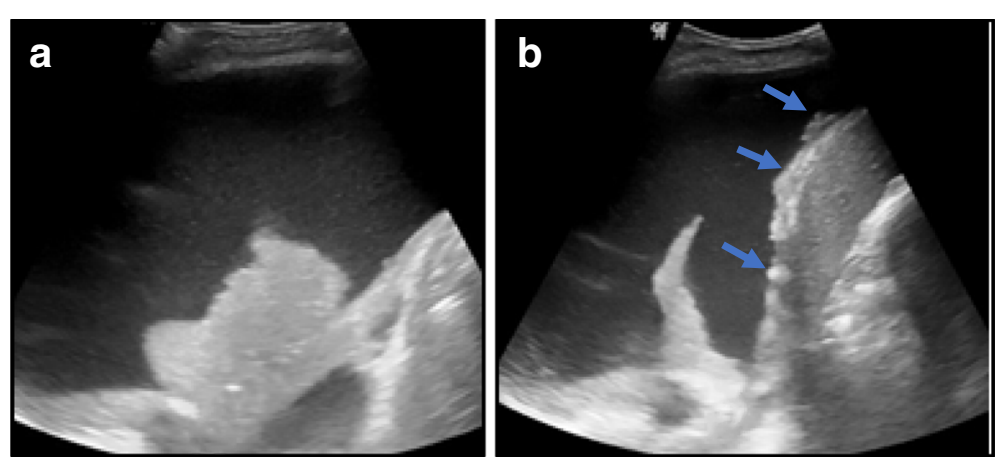

Fig. 2 US images show massive amount of free complex nonseptated pleural effusion with underlying collapsed lung (a). Note the pleural nodularity along the diaphragmatic pleura (blue arrow). Histopathology of the pleural nodules revealed necrotizing infiltrating mesothelioma 

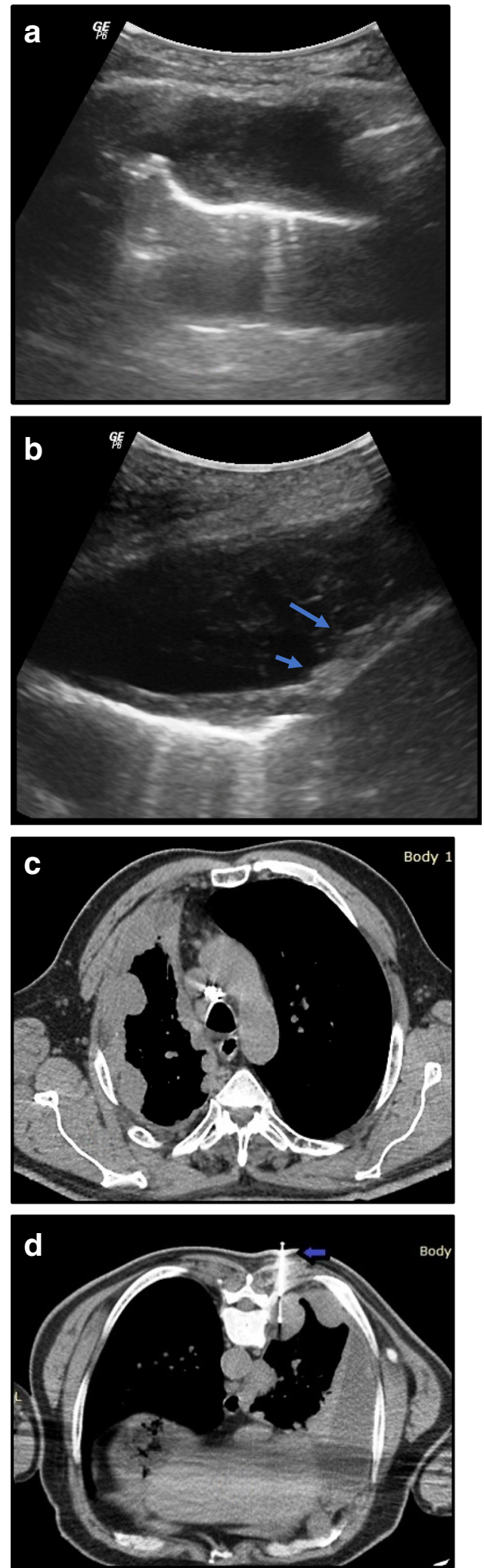

Fig. 3 US images show mild encysted complex septated pleural collection (a) with visceral pleural nodularity (blue arrows) (b, c) Non-contrasted CT chest of the same patient showed encysted pleural effusion with nodular pleural thickening and subsequent hemithoracic volume reduction. $\mathbf{d}$ CT-guided Tru-cut biopsy from one of the pleural nodules. Histopathology revealed mesothelioma

The TUS findings in both groups were correlated with the cyto-histological findings.

\section{Technique of transthoracic-guided interventional procedures}

Before the procedure, we study the CT and ultrasound images to assure the safest needle pathway to reduce the risk of complications. The procedure theater is sterilized. When the lesion has been identified, the patient is positioned comfortably, the intercostal space used as the puncture site is determined, and the depth of the lesion from the skin is measured. The puncture site is marked faintly on the skin surface, and the skin is disinfected by Betadine followed by subcutaneous local anesthetic agent injection reaching the pleural surface. The needle is inserted into the lesion under CT or US guidance, and the needle tip must be visualized within the lesion before firing the biopsy needle or aspirating the fluid. Patients are asked to hold their breath during the procedure, which usually does not exceed $10 \mathrm{~s}$ during which the sample is taken then the skin is compressed tightly by sterile dressings. About two to three cores are taken from the lesion, preserved immediately in $10 \%$ formaldehyde containers or tubes, and sent for histopathological evaluation. The aspirated pleural fluid is sent for cytological evaluation; if the result is worrisome of neoplastic process, the patient underwent thoracoscopy at the pulmonology department and pleural samples are sent for histopathological evaluation.

After the procedure, chest X-ray is done for all cases to roll out pneumothorax and the patient is hospitalized in the pulmonology department for few hours for more reassurance.

\section{Possible risk}

Risk of complications related to intervention as pneumothorax, parenchymal hemorrhage, or contusion.

\section{Statistical analysis}

Data management and statistical analysis were performed using the Statistical Package for Social Sciences (SPSS) vs. 23.

Numerical data were summarized using means and standard deviations while categorical data were summarized as numbers and percentages.

Comparisons between malignant and nonmalignant lesions were done using $X^{2}$ (chi square) test or Fisher exact 

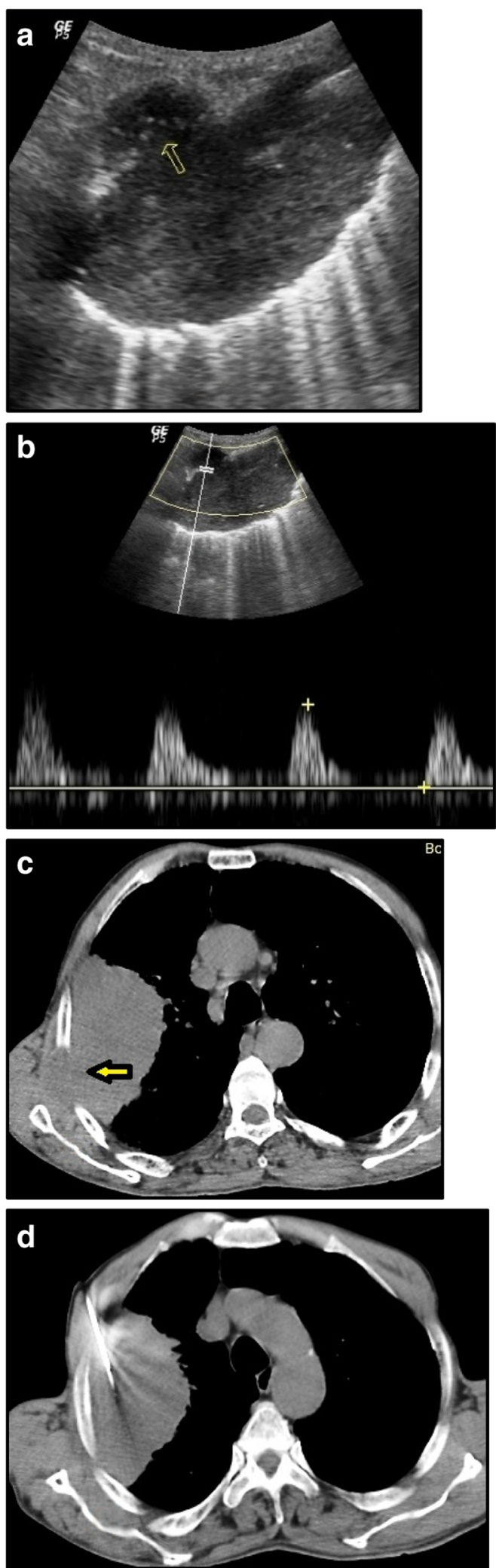

Fig. 4 US images show a well-defined isoechoic soft tissue mass lesion measuring $10 \times 6 \mathrm{~cm}$ (a) showing chest wall invasion (yellow arrow). Doppler study shows extended intralesional vascularity in the chest wall (b). Non-enhanced CT shows right-sided peripheral lung mass (c) with chest wall extension (yellow arrow). CT-guided biopsy was taken $(\mathbf{d})$. Histopathology revealed moderately

differentiated adenocarcinoma

test-if appropriate-for categorical variables. MannWhitney $U$ test was used for comparing numerical variables. Diagnostic indices including sensitivity, specificity, PPV, and NPV were calculated for ultrasound finding suggesting malignancy compared to histopathology. Overall accuracy with $95 \%$ confidence interval was calculated.

ROC analysis was done for pleural thickening in detection of malignancy. Cutoff point and diagnostic indices were calculated.

All $P$ values were two-sided. $P$ values $<0.05$ were considered significant.

\section{Results}

The results of the study sample (69 patients) was divided into group I (48 patients) including patients who presented mainly with pleural effusion and group II (21 patients) including patients who presented with peripheral pulmonary lesions.

\section{Group I (pleural effusion group)}

Forty-eight patients were enrolled within the pleural lesion group (20 males " $41.7 \%$ " and 28 females "58.3\%"). Patients' ages ranged from 15 to 73 years (mean age $=$ $50.3 \pm 15.5)$.

Histopathological/cytological evaluation of the patients in the pleural effusion group showed that 16 cases were malignant (10 males " $62.5 \%$ " and 6 females " $37.5 \%$ ") and 32 were non-malignant (10 males "31.3\%" and 22 females "68.8\%"). Within the benign cases, 24 cases revealed exudate and 8 cases revealed transudate.

According to the volume, 4 cases $(8.3 \%)$ had a minimal amount of pleural effusion, 16 cases $(33.3 \%)$ had a small amount, and 12 cases $(25.0 \%)$ had a moderate amount, while 16 cases $(33.3 \%)$ had massive amount. According to echogenicity, 18 cases $(37.5 \%)$ were clear anechoic and 18 cases $(37.5 \%)$ were complex nonseptated while 12 cases (25.0\%) were complex and septated. Also, US showed encysted effusion in 12 cases (25.0\%), pleural thickening in 18 cases (37.5\%), and underlying pulmonary consolidation/collapse in 4 cases $(8.3 \%)$.

On the correlation of the ultrasound findings with the cyto-histological results, we found that:

According to the effusion volume, 8 (50\%) of the malignant effusions were massive effusions, while only 4 cases $(25 \%)$ were small, 2 cases $(12.5 \%)$ were minimal, 
and 2 were moderate. For the benign effusions, 12 cases (37.5\%) were small, 10 (31.3\%) were moderate, 8 (25\%) were massive, and $2(6.3 \%)$ were minimal. No significant statistical difference was found between the benign and malignant lesions regarding the pleural effusion volume $(P$ value $=0.212)$.

The presence of septations within the pleural fluid also showed no significant difference between the benign and the malignant cases $(P$ value $=0.287)$ and between the inflammatory (transudate) and noninflammatory (exudate and malignant effusions) pleural effusions $(P$ value $=1)$.

Encysted pleural effusion was found in 6 malignant cases $(37.5 \%)$ and in the same number of the benign cases $(18.8 \%)$ with no significant statistical difference noted $(P$ value $=0.157)$.

Eighteen cases of the pleural effusion group showed associated pleural thickness, 8 of them were malignant and 10 were benign with no significant statistical difference in its incidence between benign and malignant lesions $(P$ value $=0.206)$. Thirteen malignant cases showed pleural nodularity in contrast to only one benign case nodularity. A significant difference was found between malignant and benign lesions regarding the presence of pleural nodularity $(P$ value $<0.001)$.

Significant statistical difference was found between the benign and malignant effusions regarding the measurement of the associated costal pleural thickening ( $P$ value $=0.021)$. Receiver operating characteristic (ROC) curve (Fig. 5) analysis was performed for the values of the pleural thickening in the benign and malignant groups. It defined cutoff value of $0.75 \mathrm{~cm}$ at a maximum combined sensitivity and specificity of $75 \%$ and $80 \%$ respectively and area under the curve of 0.825 .

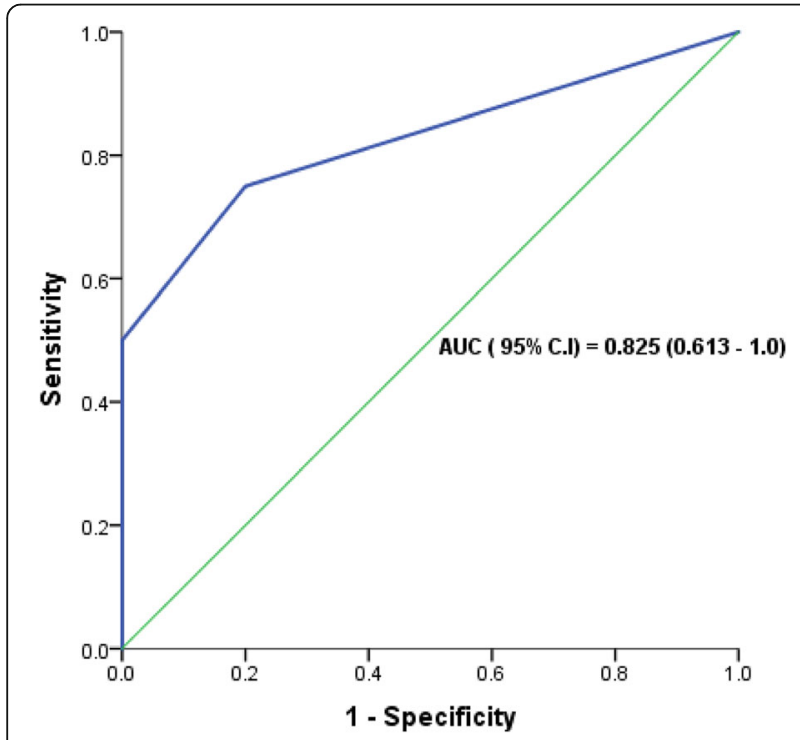

Fig. 5 ROC curve for pleural thickness values in the benign and malignant pleural effusion. Area under the curve $=0.825$

\section{Group II (peripheral pulmonary lesion group)}

Twenty-one patients were enrolled within the peripheral pulmonary lesion group (18 males $(85.7 \%)$ and 3 females $(14.3 \%))$. Patients' ages ranged from 20 to 80 years (mean age $=57.3 \pm 13.7$ ) .

In the histopathological evaluation of the pulmonary lesions, 15 cases were malignant (14 males "93.3\%" and only 1 female "6.7\%") and 6 were benign (4 males "66.7\%" and 2 females "33.3\%").

According to the mass border, 15 cases (71.4\%) had well-defined border and 6 (28.6\%) showed ill-defined one. According to echogenicity, 9 cases (42.9\%) were isoechoic and 8 (38.1\%) were hypoechoic while $4(19.0 \%)$ appeared hyperechoic. According to echopattern, 12 cases $(57.1 \%)$ were homogenous and 9 (42.9\%) were heterogeneous. According to the pattern of vascularity, 16 cases (76.2\%) showed disturbed pattern and 5 cases (23.8\%) were of normal pulmonary vascular pattern. Also, US showed intrinsic air bronchogram in 8 cases (38.1\%) and extension to the pleura in 13 cases $(61.9 \%)$ while chest wall invasion was seen in 7 cases (33.3\%).

On correlating the US findings with the cystohistological results, we found that:

According to the border definition, within the 15 malignant cases, 10 cases (66.7\%) had a well-defined border and $5(33.3 \%)$ had an ill-defined one. In the benign lesions, 5 cases $(83.3 \%)$ had a well-defined border and only 1 case $(16.7 \%)$ showed an ill-defined border. No significant statistical difference was found between the benign and malignant lesions regarding the mass border $(P$ value $=0.623$ ) .

According to the lesion echogenicity, 8 (53.3\%) of the malignant lesions were isoechoic, while 4 (26.7\%) were hypoechoic and $3(20.0 \%)$ were hyperechoic. For the benign lesions, 4 (66.7\%) were hypoechoic while only 1 case $(16.7 \%)$ was isoechoic and the other one $(16.7 \%)$ was hyperechoic. No significant statistical difference was found between the benign and malignant lesions regarding the pulmonary mass echogenicity $(P$ value $=0.211$ )

According to the lesion echopattern, 8 malignant cases (53.3\%) and 4 benign cases (66.7\%) were homogenous, while 7 malignant cases (46.7\%) and only 2 benign cases $(33.3 \%)$ were heterogeneous. No significant statistical difference was found between the benign and malignant lesions regarding lesion echopattern $(P$ value $=0.659)$.

According to the intralesional air bronchogram, 3 (20\%) of 15 malignant lesions while 5 (83.3\%) of 6 benign lesions showed intrinsic air bronchogram. A significant difference was found between the benign and malignant lesions regarding the intralesional air bronchogram $(P$ value $=0.014)$.

Intralesional disrupted vascularity pattern was found in all the malignant lesions (100\%), while only it was found in one benign case (16.7\%). It showed a 
significant difference between malignant and benign lesions $(P$ value $\leq 0.001)$.

Seven (46.7\%) of the malignant lesions showed an extension to the pleura or the chest wall while none of the benign lesions showed evidence to the pleural or chest wall invasion. There was a significant difference between malignant and benign lesions regarding the pleural $(P$ value $=0.016)$ and chest wall $(P$ value $=0.004)$ invasion respectively.

\section{Discussion}

According to Nance et al., pleural fluid cytology was not able to establish diagnosis of malignant pleural effusion in $40 \%$ of their study cases [14]; thus, in our study, nearly all the cases underwent Tru-cut or surgical biopsy and histopathology-except 3 patients in the pleural effusion group who underwent pleural effusion cytology and aspiration only.

In our study, we found that pleural effusion volume and encystation were not differentiating factors between benign and malignant pleural effusion. We agreed with Chen et al. [15], Quershi et al. [6], and Yang et al. [11] that the septated complex pattern is not significant in differentiating malignant from benign pleural effusions. We also postulated that a septated complex pattern cannot differentiate between inflammatory and noninflammatory pleural effusions, while Chen et al. stated that the septated complex pattern is a good predictor for tuberculosis in the lymphocyte-rich exudative pleural effusion [15].

According to Bugalho et al. [10] and Quershi et al. [6], pleural nodularity and involvement of diaphragmatic pleura were good predictors of malignancy with specificity of $91-100 \%$ and sensitivity of $78-73 \%$ respectively. We agreed with them as pleural nodularity was significant for malignancy ( $P$ value $<0.001)$. Also, we went with Bugalho et al. who stated that pleural nodularity is not pathognomonic for malignancy, as it was also seen in tuberculous effusions in their study [10].

Quershi et al. [6] and Bugalho et al. [10] agreed together that pleural thickening $>10 \mathrm{~mm}$ is a predictor of malignancy. Our study showed little difference regarding the cutoff value of the pleural thickening, being $7.5 \mathrm{~mm}$ with maximum combined sensitivity and specificity of $75 \%$ and $80 \%$ respectively.

The circumferential pleural involvement and involvement of the mediastinal pleura were among the CT predictive parameters of malignancy according to Yilmaz et al. [7] and Leung et al. [16]. These factors cannot be assessed by TUS due to the narrow acoustic window and the limited penetration, thus considered one of the weak points of the TUS against CT.

In the peripheral pulmonary lesions, we found that the presence of intralesional air bronchogram is statically significant for benign lesions. Our findings are supportive to Bugalho et al. [10] who stated that the absence of intralesional air bronchogram is a suggestive sign of malignancy with high sensitivity of $92.4 \%$.

Also, we found that intralesional vascular distortion is a significant sign of malignancy. So, we agreed with Rednic et al. [1] who postulated that bronchial and vascular distortion is a differentiating sign between malignant and benign lesions, while we partially disagreed with Abd El-hafez et al. [9] who found that destruction of the normal tissue architecture and blood vessel displacement are more common (but not statistically significant) in the malignant rather than in the benign group.

On the other hand, we did not go with Rednic et al. [1] in his conclusion that regular lesion outline was suggestive of malignancy while irregular and saw tooth-like lesion borders were suggestive of benign lesions, as we found no statistical significance between benignity and malignancy regarding the lesion border.

Because Suzuki et al. [17] and Bandi et al. [18] found that US is superior to the CT in the evaluation of chest wall invasion, so we studied pleural and chest wall invasion as a differentiating factor between benign and malignant lesions.

Our results agreed with that of Bugalho et al. [10] who stated that signs of pleural invasion as pleural surface reflection and signs of chest wall invasion as tumor fixation during breathing, extension of the tumor through the chest wall, and rib invasion are highly specific for malignancy. Also, Rednic et al. [1] found that the irregular overlying lung outline is a suggestive sign of malignancy while a regular outline went with benignity. All of these results are against Abd El-hafez et al. [9] who found that pleural nodularity was not significant for malignancy lesions and more often seen with tuberculosis.

The limitations of this study include that it was held out in a single hospital, there were a relative small number of the subjects within each group, there was a lack of long-term follow-up for the diagnosed benign cases (for further confirmation of diagnosis considering histopathological bias), and the definite diagnosis depends only on the cytological findings in 3 cases of our study sample.

So we recommended that the points of disagreement between us and the previous works are to be studied over larger number of cases to verify our findings.

\section{Conclusion}

TUS is a nonconclusive diagnostic tool in differentiation between benign and malignant pleural effusion, yet the presence of pleural nodularity and costal pleural thickening $>7.5 \mathrm{~mm}$ are predictors of malignancy. Also, distorted intralesional vascular pattern and pleural and chest wall invasion are strong ultrasonographic 
malignant predictors in the peripheral pulmonary lesions. TUS cannot replace CT in the evaluation of pleural and peripheral pulmonary lesions due to the narrow acoustic window and inability to examine deeper structures; however, it is a valuable, safe, cheap, and easily accessible complementary modality in the evaluation of just cases.

\section{Abbreviations}

CT: Computed tomography; NPV: Negative predictive value; PPV: Positive predictive value; ROC: Receiver operating characteristics; SPSS: Statistical Package for Social Sciences; TUS: Transthoracic ultrasound; UP: Ultrasound pattern; US: Ultrasound

\section{Acknowledgements}

I would like to thank Dr. Sara Mansour for her sincere help in this work.

\section{Author's contributions}

The author read and approved the final manuscript.

\section{Funding}

The study was not funded by agencies.

\section{Availability of data and materials}

The datasets used and/or analyzed during the current study are available from the corresponding author on reasonable request.

\section{Ethics approval and consent to participate}

A written informed consent was taken from all the subjects. Ethical consideration had been approved by the ethical committee of Banha Faculty of Medicine.

\section{Competing interests}

The author declares that he has no competing interests.

Received: 6 June 2019 Accepted: 2 July 2019

Published online: 05 August 2019

\section{References}

1. Rednic N, Orasan O (2010) Subpleural lung tumors ultrasonography. Med Ultrason 12(1):81-87

2. Trovato GM, Sperandeo M, Catalano D (2014) Optimization of thoracic US guidance for lung nodule biopsy. Radiology 270(1):308-308

3. Vorster MJ, Allwood BW, Koegelenberg CF (2015) Transthoracic ultrasonography for clinicians. Eurasian J Pulmonol 17(1):1-9

4. Bugalho A, Semedo J, Alpendre J, Cepeda Ribeiro J, Carreiro L (2010) Ultrasound in chest disease. Rev Port Pneumol 16(4):589-606

5. Hallifax RJ, Corcoran JP, Ahmed A, Nagendran M, Rostom H, Hassan N et al (2014) Physician-based ultrasound-guided biopsy for diagnosing pleural disease. Chest 146(4):1001-1006

6. Qureshi NR, Rahman NM, Gleeson FV (2009) Thoracic ultrasound in the diagnosis of malignant pleural effusion. Thorax 64(2):139-143

7. Yilmaz U, Polat G, Sahin N, Soy O, Gülay U (2005) CT in differential diagnosis of benign and malignant pleural disease. Monaldi Arch Chest Dis Arch Monaldi Mal Torace 63(1):17-22

8. Hierholzer J, Luo L, Bittner RC, Stroszczynski C, Schröder RJ, Schoenfeld N et al (2000) MRI and CT in the differential diagnosis of pleural disease. Chest 118(3):604-609

9. Elhafez SAMA, Shehata MEA, Rashed MAE, Ali RE, Mosallam AMA (2013) Echopulmonography versus computed tomographic chest predictors for differentiation between benign and malignant peripheral pulmonary and pleural lesions. Egypt J Chest Dis Tuberc 62(1):127-135

10. Bugalho A, Ferreira D, Dias SS, Schuhmann M, Branco JC, Marques Gomes MJ et al (2014) The diagnostic value of transthoracic ultrasonographic features in predicting malignancy in undiagnosed pleural effusions: a prospective observational study. Respiration 87(4):270-278

11. Yang PC, Luh KT, Chang DB, Wu HD, Yu CJ, Kuo SH (1992) Value of sonography in determining the nature of pleural effusion: analysis of 320 cases. AJR Am J Roentgenol 159(1):29-33
12. Tsai T-H, Yang P-C (2003) Ultrasound in the diagnosis and management of pleural disease. Curr Opin Pulm Med 9(4):282-290

13. Sugama Y, Tamaki S, Kitamura S, Kira S (1988) Ultrasonographic evaluation of pleural and chest wall invasion of lung cancer. Chest 93(2):275-279

14. Nance KV, Shermer RW, Askin FB (1991) Diagnostic efficacy of pleural biopsy as compared with that of pleural fluid examination. Mod Pathol Off J U S Can Acad Pathol Inc 4(3):320-324

15. Chen H-J, Hsu W-H, Tu C-Y, Yu Y-H, Chiu K-L, Hang L-W et al (2006) Sonographic septation in lymphocyte-rich exudative pleural effusions: a useful diagnostic predictor for tuberculosis. J Ultrasound Med Off J Am Inst Ultrasound Med 25(7):857-863

16. Leung AN, Müller NL, Miller RR (1990) CT in differential diagnosis of diffuse pleural disease. AJR Am J Roentgenol 154(3):487-492

17. Suzuki N, Saitoh T, Kitamura S (1993) Tumor invasion of the chest wall in lung cancer: diagnosis with US. Radiology 187(1):39-42

18. Bandi V, Lunn W, Ernst A, Eberhardt R, Hoffmann H, Herth FJF (2008) Ultrasound vs. $C T$ in detecting chest wall invasion by tumor: a prospective study. Chest 133(4):881-886

\section{Publisher's Note}

Springer Nature remains neutral with regard to jurisdictional claims in published maps and institutional affiliations.

\section{Submit your manuscript to a SpringerOpen ${ }^{\circ}$ journal and benefit from:}

- Convenient online submission

- Rigorous peer review

- Open access: articles freely available online

- High visibility within the field

- Retaining the copyright to your article

Submit your next manuscript at $\boldsymbol{\nabla}$ springeropen.com 